\title{
Reversible Thermal Gradient Device to Control Biased Thermotactic Response of C. elegans
}

\author{
Jin-Sung Park, Geunseob OH, Jiwon KIM, Eun Young PARK, and Jennifer H. SHIN ${ }^{\dagger}$ \\ Department of Mechanical Engineering, Korea Advanced Institute of Science and Technology (KAIST), \\ 291 Daehak-ro, Yuseong-gu, Daejeon 34141, Korea
}

\begin{abstract}
C. elegans exhibits a directional migration toward a remembered temperature setpoint $\left(T_{\mathrm{s}}\right)$ by activating thermosensorimotor neurons. While cryophilic thermotaxis is well reproduced, thermophilic thermotaxis requires very stringent temperature regulations - otherwise, worms exhibit random migration in colder side of $T_{\mathrm{s}}$. Here, we introduce a thermal stimulus device developed to control worms with different thermotactic behaviors on both colder and warmer sides of the $T_{\mathrm{s}}$. On a linear gradient, the worm population displayed a Gaussian distribution near $T_{\mathrm{s}}$ but in a skewed shape with a peak shifted to the colder side due to their atactic motion in colder temperature than $T_{\mathrm{s}}$. By repetitive application of thermal gradient-reversals, we found that their population density became higher near $T_{\mathrm{s}}$ because the speed at which the worms accumulate toward $T_{\mathrm{s}}$ was much faster than that of the dispersion by diffusion to the cold side, resulting in forced aggregation of worms at the desired temperature.
\end{abstract}

Keywords C. elegans, thermotaxis, gradient-reversal, thermal stimulus, forced aggregation

(Received May 24, 2019; Accepted August 20, 2019; Advance Publication Released Online by J-STAGE August 30, 2019)

\section{Introduction}

The nematode C. elegans has served as an excellent model organism in various behavioral modes of sensory responses to multiple exogenous stimuli. These worms actively alter their locomotion gait, ${ }^{1,2}$ stroke shape, ${ }^{3,4}$ and navigation strategy ${ }^{5-7}$ in response to changes in various environmental factors for their survival. As one of the most popular navigation strategies, C. elegans can direct their migration, termed "taxis", toward or away from exogenous cues of chemical, ${ }^{8,9}$ electrical, ${ }^{10,11}$ optical, ${ }^{12}$ or thermal perturbations. ${ }^{13-17}$

When a thermal gradient is generated in space, C. elegans senses a temperature difference across their body in contact with the environment and directs their migration to find the preregistered temperature setpoint $\left(T_{\mathrm{s}}\right)$, determined from the retrospective memory for the past cultivation temperature. To fully register the specific temperature value as $T_{\mathrm{s}}$, the worms need to be exposed to a fixed temperature in the range of 15 to $25^{\circ} \mathrm{C}$ for at least $4 \mathrm{~h}^{6}{ }^{6,13,15,17}$ According to the previous studies, C. elegans exhibits both cryophilic and thermophilic thermotaxis, classified by the gradient direction where worms migrate in the direction parallel to the thermal gradient. ${ }^{18-21}$ Cryophilic thermotaxis is observed in the worms placed on the warm temperature side $\left(>T_{\mathrm{S}}\right)$ on a linear thermal gradient, where they migrate along the negative gradient to seek $T_{\mathrm{s}}$. While the cryophilic thermotaxis can be reproduced in a broad range of temperature conditions in laboratory settings, the thermophilic thermotaxis requires very strict temperature conditions including (i) setting the value of $T_{\mathrm{s}}$ over $23^{\circ} \mathrm{C}$, and (ii) exposing to a

$\dagger$ To whom correspondence should be addressed.

E-mail: j_shin@kaist.ac.kr shallow thermal gradient, $\Delta T_{\text {steep }}<0.5^{\circ} \mathrm{C} / \mathrm{cm}$, within $5^{\circ} \mathrm{C}$ compared to $T_{\mathrm{s}}$ (Fig. 3C). ${ }^{6,21,22}$ Otherwise, instead of a thermotactic behavior, the worms exhibit a random migration on the cold temperature side $\left(<T_{\mathrm{s}}\right)$, similar to the behavior of a worm migrating on an isothermal plate.

Compared to other tactic behaviors, thermotaxis in C. elegans is of particular interest because it provides the opportunity for better understanding of the relationship between the establishment of long-term memory and behavioral responses as an output. Traditionally, the water-reservoir typed thermal gradient device was commonly used to study C. elegans thermotaxis. . $^{13,20,21}$ In this type, a linear thermal gradient was established across an agar plate placed on a conducting metal plate, bridging across the hot and cold reservoirs where each temperature is maintained continuously by circulating temperature-controlled water from two pumps. Recently, the massive water reservoirs were replaced by the Peltier module and the resistive cartridge heater for cooling and heating, respectively. ${ }^{6}$ Nevertheless, it was still complicated to equip this device onto a microscopic stage because a water-coolant type of the Peltier device was used for heat removal. Also, the direction of a linear gradient was fixed as the cartridge heater could be only operated in a heating mode.

While there have been many attempts to develop various forms of specifically designed micro-fluidic chips for different sources of stimuli to investigate the intrinsic tactic-behaviors of C. elegans, $, 911,23-25$ the practical realization of a thermal gradient has been challenging due to their atactic response in a thermophilic direction. Therefore, it will be conducive to develop a thermal stimulus device that can effectively control bi-directional movements of worms for systematic investigation of their thermotactic responses. In this study, we introduce a new type of a linear thermal gradient device that can apply a 
thermal stimulus of gradient-reversal to aggregate worms at a high population near $T_{\mathrm{s}}$ by controlling their migration bidirectionally. As the gradient-reversal is repeatedly applied, the population density of worms near $T_{\mathrm{s}}$ becomes higher as their accumulation speed toward $T_{\mathrm{s}}$ is much faster than diffusion to the cold side. Our experimental results verify that the gradientreversal stimulus device could be used as an external triggering source to selectively collect worms with rapid responsiveness to a temperature shock.

\section{Experimental}

\section{Design of a Peltier-based thermal gradient device}

The developed thermal gradient device is divided into three major parts: the Peltier module, thermal conducting plate, and PID-feedback system (Fig. 1A)

A core of the Peltier module (Fig. 1B) is a thermoelectric semiconductor device (dimension: $30 \times 30 \times 3.8 \mathrm{~mm}, 26 \mathrm{~W}$ TEC1-07105T125, Hebei, Shanghai). By applying a DC voltage, the Peltier element pumps heat from its one side to the other side. As a temperature difference between both ends increases, a natural heat flow by heat conduction between both sides passively arises from the hot to the cold side. Because a passive heat flow works against the active Peltier heat pumping, it lowers the Peltier performance. To suppress a passive heat flow, a plate-fin type heat sink $(71 \times 100 \times 30 \mathrm{~mm}$, aluminum $)$ with an electric fan $(70 \times 70 \times 15 \mathrm{~mm}, 70 \mathrm{~W}$ Apachi deepcool $\mathrm{X}$-fan) was attached to one side of the Peltier element to release heat from the Peltier element into the ambient air. Then, the heat conducting metal $(46(\mathrm{~W}) \times 30(\mathrm{~L}) \times 5(\mathrm{H}) \mathrm{mm}$, aluminum $)$ with a projecting part $(30(\mathrm{~W}) \times 10(\mathrm{~L}) \times 5(\mathrm{H}) \mathrm{mm})$ was attached to the opposite side of the Peltier element to enhance a heat transfer efficiency to the agar block. Here, its projecting part was in contact with one end side of the agar block through a square-shaped hole on the side of the outer agar block case for heat insulation. The remaining surfaces of the Peltier element sandwiched between a plate-fin type heat sink and a heat conducting metal were covered with Styrofoam (thermal conductivity $(\kappa)$ of $0.03 \mathrm{~W} / \mathrm{m} \cdot \mathrm{K}$ ) for insulation. Basically, two Peltier modules attached to the agar block have the same structure, but each operated in the opposite polarity to each other: thus, one functions as a heat source and the other as a heat sink.

The second part is the thermally conducting plate composed of a soft agar block (1.7\%, A1296, Sigma, USA) and its outer insulating case (Fig. 1C). In the laboratory, an agar as a biologically inert and viscoelastic material provides an inhabited environment for $C$. elegans. The insulating case made of a transparent polycarbonate material $(46(\mathrm{~W}) \times 49(\mathrm{~L}) \times 30(\mathrm{H})$ mm, thickness: $8 \mathrm{~mm}$ with $\kappa \sim 0.19 \mathrm{~W} / \mathrm{mK}$ ) allows microscopic imaging in a transmitted light mode. Through the square-shaped holes $(30 \times 12 \mathrm{~mm})$ in both ends, the projecting parts in two heat conducting metals are tightly fitted. For temperature measurements, a total of seven holes (diameter, $\phi \sim 2.0 \mathrm{~mm}$ ) were punched in one side of the outer insulating case to pin the platinum resistance temperature detectors (Pt-RTDs, $\phi \sim 1.8 \mathrm{~mm}$ ) with a tolerance of $(0.6+0.008 T){ }^{\circ} \mathrm{C}$ at $5 \mathrm{~mm}$ intervals. Among them, both end holes were used for Pt-RTDs to send input signals to PID controllers.

The last part is a proportional-integral-derivative (PID) feedback system for temperature control. A PID controller is the most common form of feedback, which has been widely used in various industrial applications. In our device, it consisted of a conventional PID controller (SDU440, Sanup
A

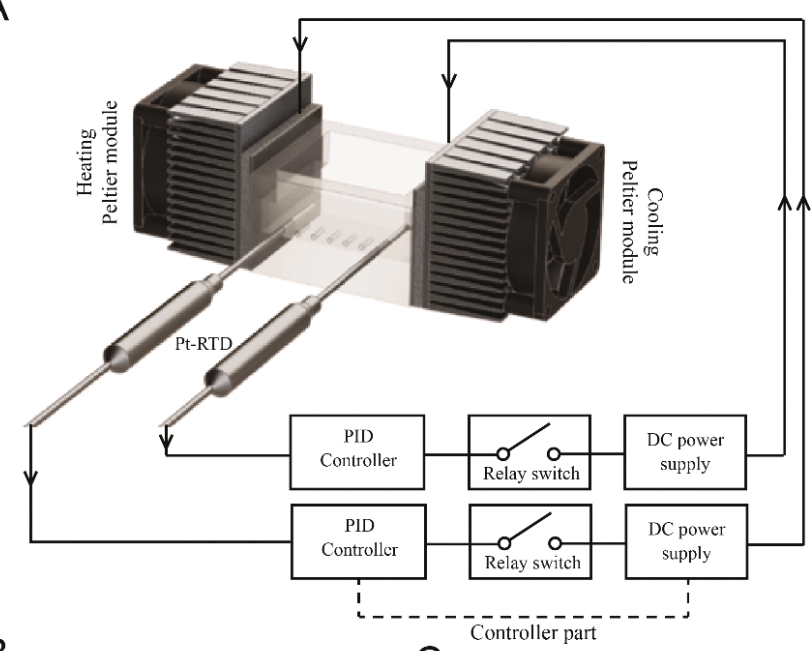

$B$

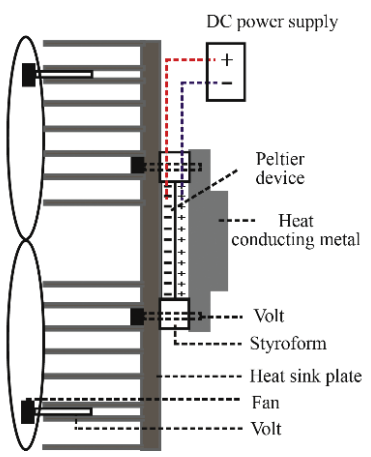

C

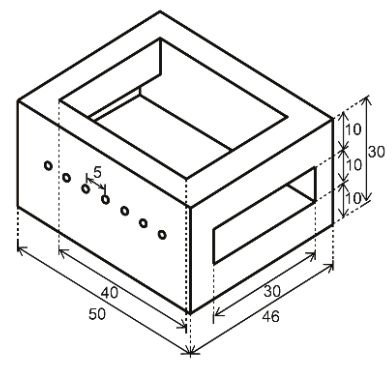

Fig. 1 Schematic design of a Peltier-based thermal gradient apparatus developed for the study of thermotaxis in C. elegans: (A) A thermal gradient apparatus composed of three major parts including two Peltier modules, a heat conducting plate, and two PID-feedback control systems. (B) An assembled structure of the Peltier module in detail. Here, the Peltier device is sandwiched between a heat conducting metal and a heat sink plate with an electric fan. (C) A 3-dimensional view of an outer case made of PC material for heat insulation. There are two rectangular holes for inserting the projection part in a heat conducting metal of the Peltier module. Also, a total of seven holes were punched to pin Pt-RTDs for temperature measurements and to send input signals to each PID controller. In (C), the unit in length scale is $\mathrm{mm}$.

Electric Co., Ltd., Korea), solid state relay switch (FSD 2416, Fine Suntronics, Korea), and DC power supply (GPS-3303, GW Instek, China) (Fig. 1A). After receiving the input signals from two Pt-RTDs, each PID controller sends output signals to the relay switches to control the temperature values at both end sides of an agar block in On-Off mode.

\section{Computational simulations on the formation of a spatial linear gradient across the agar block}

Thermal gradient established across an agar block was tested by simulation using the heat transfer module in COMSOL Multiphysics. Each temperature at both ends of an agar block was fixed to 8 and $42^{\circ} \mathrm{C}$. Thus, $\Delta T_{\text {diff }}$ was $36^{\circ} \mathrm{C}$. The $\kappa$ values of the agar block and its insulating case were 0.638 and $0.19 \mathrm{~W} / \mathrm{m} \cdot \mathrm{K}$, respectively. For the upper side of an agar block, two different boundary conditions were considered: the perfect insulation and the heat loss condition by heat conduction to the ambient air environment at $22^{\circ} \mathrm{C}$. For other sides of an agar block except for the upper side, the heat was transferred to the outer insulating case by natural heat convection. 
A

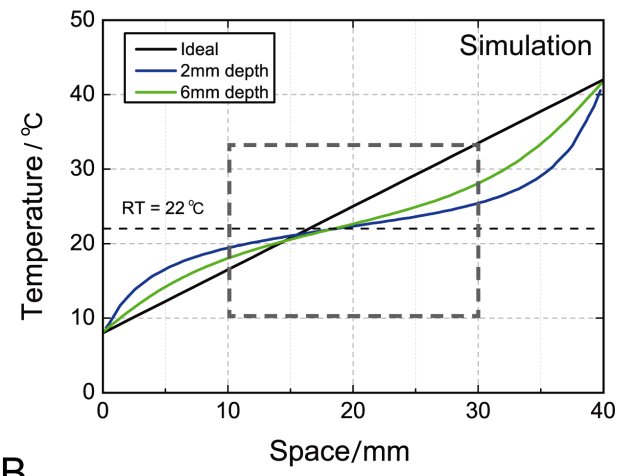

B

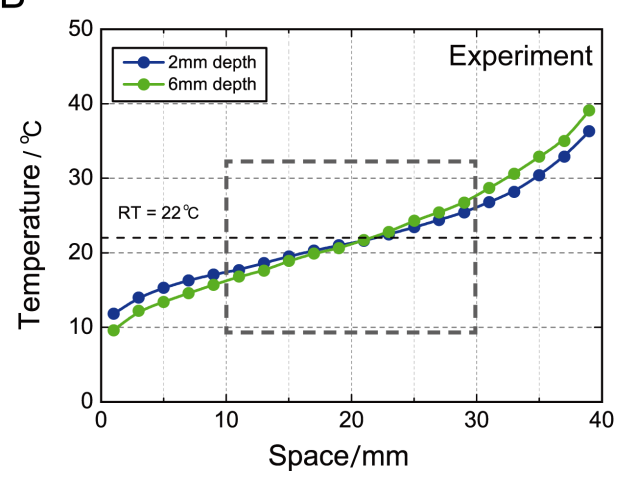

Fig. 2 The features of a spatial thermal gradient formed across an agar plate for a variation of surface depth: (A) Computational results showing the spatial shape of a thermal gradient calculated from two different boundary conditions for the upper side of an agar plate [black: the ideal case of a perfect insulation, blue and green: the cases of a natural thermal conduction with the ambient air environment, each calculated at the locations of 2 and $6 \mathrm{~mm}$ depth, respectively]. (B) Experimental data measured at the locations of 2 and $6 \mathrm{~mm}$ depth at $2 \mathrm{~mm}$ intervals. In (A) and (B), the fixed temperature values at both sides were given as 8 and $42^{\circ} \mathrm{C}$. Thus, the $\Delta T_{\text {diff }}$ was $34^{\circ} \mathrm{C}$ at the ambient air temperature of $22^{\circ} \mathrm{C}$. The linear regions of a thermal gradient between 10 and $30 \mathrm{~mm}$ were each indicated by the dotted boxed area in (A) and (B).

\section{Optical imaging of thermotactic C. elegans}

Young adult wild-type (N2) C. elegans (body length: $\sim 950 \mu \mathrm{m}$ ) were used for our experiments (Fig. S1 in Supporting Information). Before placing worms on the surface of an agar block, they were pre-incubated on the OP50-seeded NGM plate for $12 \mathrm{~h}$ at $22^{\circ} \mathrm{C}$ to set this temperature as $T_{\mathrm{s}}$ (for more details, see the section of "C. elegans preparation" in Supporting Information). A monochrome CCD camera (Flea3-U3-13Y3M, Point grey, USA) mounted on a stereo-microscope (SMZ800, Nikon, Japan) was used for recording thermotactic behaviors of C. elegans in a time-lapse mode at $1 \mathrm{~Hz}$. For manual positioning and tracking, a thermal gradient device was equipped onto the $\mathrm{X}$-Y microscope stage placed on a stereo-microscope. Each trajectory for individual worms crawling on an agar surface was analyzed using ImageJ software (NIH, USA) after recording all image sequences.

\section{Results and Discussion}

Development of a thermal gradient on an agar block

Figure 2A shows the simulation results of the spatial features in thermal gradients established across an agar block calculated
A

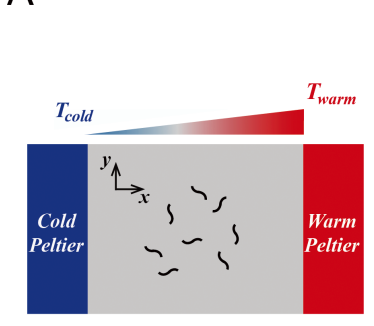

B

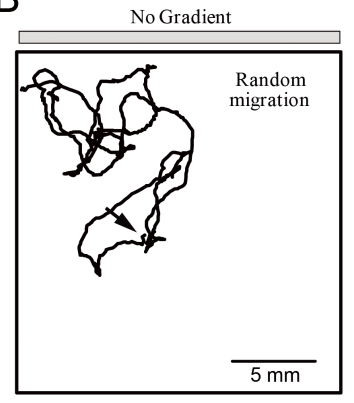

C
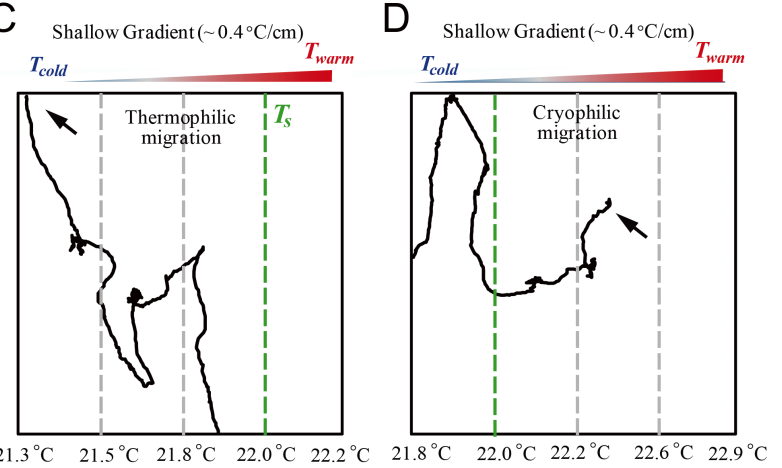

E

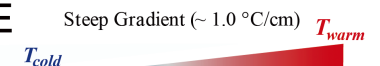

F Steep Gradient $\left(\sim 1.0^{\circ} \mathrm{C} / \mathrm{cm}\right) T_{\text {warm }}$
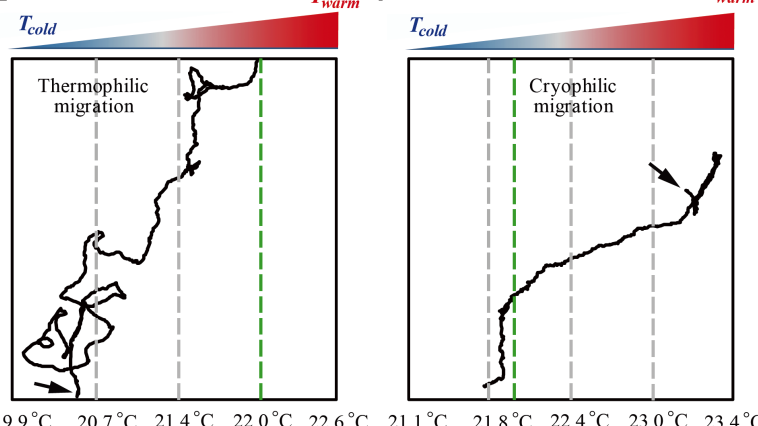

Fig. 3 The representative thermotactic behaviors of C.elegans observed in a shallow and steep thermal gradient formed across an agar surface: (A) Schematic up-side view of worms placed on an agar block sandwiched between two Peltier modules, each operated in a heating and cooling mode. (B) A typical trajectory of a worm crawling on an isothermal space. (C, D) A weakly-biased thermophilic (C) and a cryophilic (D) migration of a worm toward $T_{\mathrm{s}}$ on a space with a shallow gradient $\left(\sim 0.4^{\circ} \mathrm{C} / \mathrm{cm}\right)$. (E, F) A random-like migration of a worm observed on the side of cold temperatures $\left(<T_{\mathrm{s}}\right)(E)$ and a cryophilic migration on the side of warm temperatures $\left(>T_{\mathrm{s}}\right)(\mathrm{F})$ on a space with a steep thermal gradient $\left(\sim 1.0^{\circ} \mathrm{C} / \mathrm{cm}\right)$. In $(\mathrm{B}-\mathrm{F})$, each trajectory was recorded for $16,12,9,12$, and $10 \mathrm{~min}$ at $1 \mathrm{~Hz}$, respectively, and each starting point in the trajectory paths was indicated by the arrows. Here, the locations of $T_{\mathrm{s}}$ were indicated by green dotted lines in $(\mathrm{C}-\mathrm{F})$.

from two different depth locations $(d)$ of 2 and $6 \mathrm{~mm}$ (here, $d=0$ at the surface of an agar block). Assuming the boundary condition of perfect insulation for the upper side, the linear thermal gradients are established by connecting two fixed temperatures at each end (black lines in Fig. 2A). Also, the profiles at $d=2$ and $6 \mathrm{~mm}$ precisely overlapped with each other.

On the other hand, with the boundary condition of natural heat transfer, spatial gradients appear to be hyperbolic as shown by the blue and green lines for $d=2$ and $6 \mathrm{~mm}$, respectively (Fig. 2A). Although the slope of thermal gradients is changed immediately near both ends, they remain linear in the middle region of 10 to $30 \mathrm{~mm}$ in the agar block (boxed region in 
A
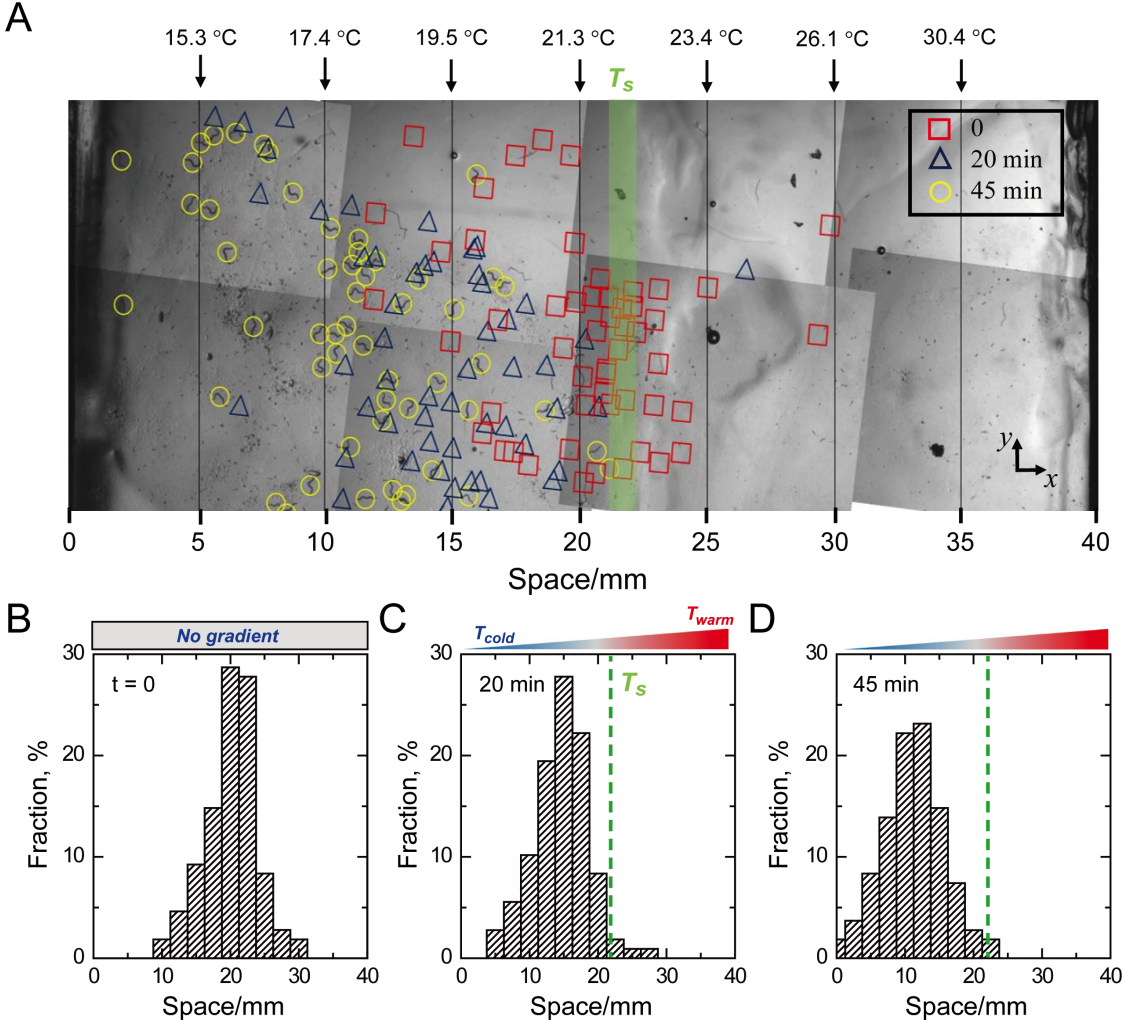

Fig. 4 Cryophilic and athermotactic behavior of $C$. elegans on the agar surface with a large value in $\Delta T_{\text {steep }}$ : (A) The snapshot image of a total of 45 worms crawling on the thermal gradient plate obtained at $t=45 \mathrm{~min}$. The locations of worms at three different $t$ are each marked by different symbols (rectangular: 0, triangles: $20 \mathrm{~min}$, and circles: $45 \mathrm{~min}$ ). (B - D) Sliding window histogram showing the peak shift in distributions of worms migrating on the agar surface over $t$. In histograms, it was measured at $2.5 \mathrm{~mm}$ intervals with a window size of $5 \mathrm{~mm}$, and the $\Delta T_{\text {steep }}$ measured between 10 and $30 \mathrm{~mm}$ was about $4.3^{\circ} \mathrm{C} / \mathrm{cm}$. In (A), each temperature value was measured at the depth of $2 \mathrm{~mm}$ at $5 \mathrm{~mm}$ intervals from the agar surface, and the green area is the temperature zone corresponding to $T_{\mathrm{s}}=22^{\circ} \mathrm{C}$. Also, see another experimental test reproduced in the same temperature conditions (Fig. S4 in Supporting Information).

Fig. 2A). Because the effect of a heat loss to the ambient air is most dominant at the interface between the agar surface and the air, the $\Delta T_{\text {steep }}$ in the linear region becomes shallower as it reaches closer to the agar surface. Based on our calculations, the values of $\Delta T_{\text {steep }}$ measured in a linear regime between 10 and $30 \mathrm{~mm}$ are about 3.5 and $4.5^{\circ} \mathrm{C} / \mathrm{cm}$ at $d=2$ and $6 \mathrm{~mm}$, respectively.

Next, we compare the experimental data measured from the Peltier-based thermal gradient device to the simulation results. In 15 min after applying DC powers to both Peltier modules, a linear gradient reached a steady state (Fig. S2A in Supporting Information). In all experiments, the temperature measurements were obtained by vertically inserting a pt-RTD probe into the agar block to the locations of $d=2$ and $6 \mathrm{~mm}$ at $2 \mathrm{~mm}$ intervals (blue and green line in Fig. 2B, respectively). Consistently with the simulation results performed with the boundary condition of natural heat loss to ambient air (blue and green lines in Fig. 2A), the profile of the thermal gradient was hyperbolic in $d=2$ and $6 \mathrm{~mm}$ (blue and green lines in Fig. 2B, respectively). According to our measurements, each value in $\Delta T_{\text {steep }}$ measured in a linear region of 10 to $30 \mathrm{~mm}$ was about 4.3 and $5.5^{\circ} \mathrm{C} / \mathrm{cm}$ in $d=2$ and $6 \mathrm{~mm}$ at the ambient air temperature of $\sim 22^{\circ} \mathrm{C}$, respectively, showing good consistency with the simulation results.
Thermotactic behaviors of C. elegans on a steep thermal gradient plate

Figure 3 shows the typical thermotactic behaviors of C. elegans reproduced in our developed thermal gradient device (Fig. 3). In the absence of a thermal gradient, a worm exhibited a random migratory behavior, which is characterized by a straight movement followed by random events of sharp and shallow turns (Fig. 3B and Fig. S3 in Supporting Information). Given a linear thermal gradient on an agar surface, the cryophilic thermotaxis appeared in both cases of a shallow $\left(\Delta T_{\text {shallow }}\right.$ $\left.\sim 0.4^{\circ} \mathrm{C} / \mathrm{cm}\right)$ and steep temperature gradient $\left(\Delta T_{\text {steep }} \sim 1.0^{\circ} \mathrm{C} / \mathrm{cm}\right)$ (Figs. 3D and 3F, respectively). Meanwhile, the thermophilic thermotaxis for the cultivated worms at $22^{\circ} \mathrm{C}$ showed a weaklybiased migration toward $T_{\mathrm{s}}$ in a shallow gradient (Fig. 3C). In the steep gradient, the overall migration behavior of worms appeared random as shown in Fig. 3B (Fig. 3E). Also, the isothermal tracking in $C$. elegans thermotaxis was observed in a shallow thermal gradient $\left(\Delta T_{\text {shallow }} \sim 0.4^{\circ} \mathrm{C} / \mathrm{cm}\right)$, showing a rectilinear migration along a narrow isothermal area of $T_{\mathrm{s}}$ (Fig. S4 in Supporting Information). ${ }^{6,13,15,26}$

To verify the performance of the reversible thermal gradient device as a stimulus device for aggregating worms, we first examined the thermotactic behaviors in the population of worms in a steeper thermal gradient with $T_{\text {steep }} \sim 4.3^{\circ} \mathrm{C} / \mathrm{cm}$. After preincubation at $22^{\circ} \mathrm{C}$ for $12 \mathrm{~h}$, these worms were plated around 
A

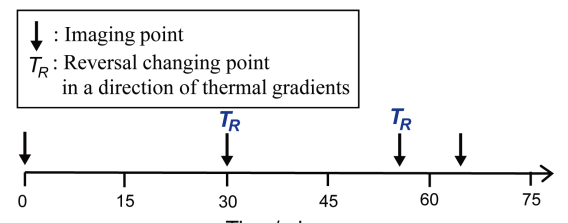

B
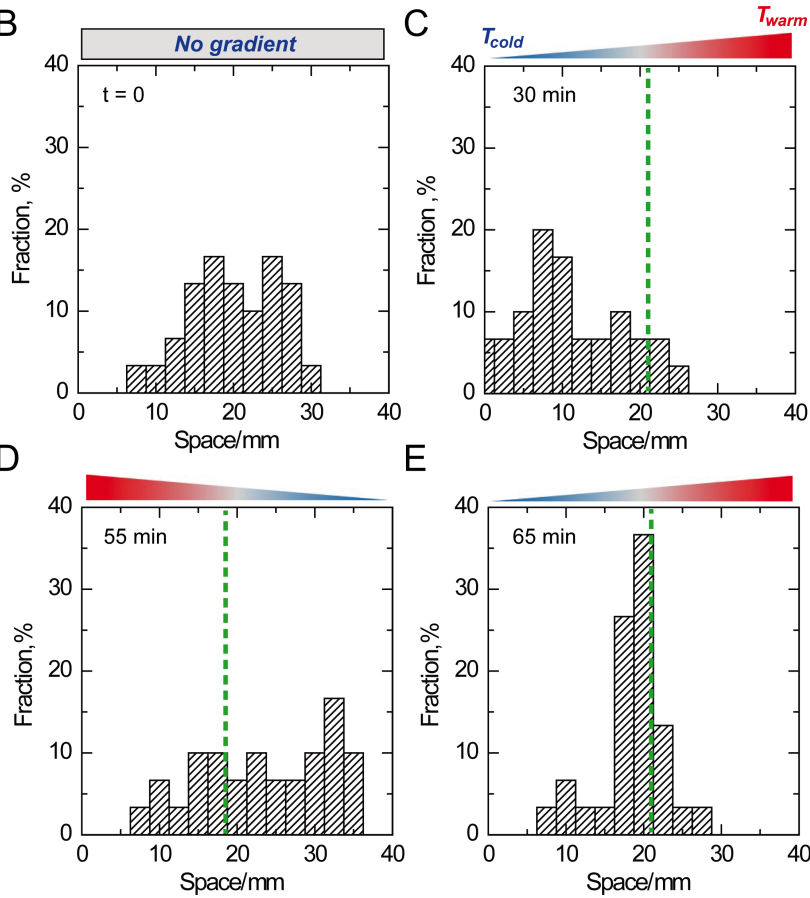

Fig. 5 Aggregation in the population of C. elegans induced by a reversal change in the direction of thermal gradients: (A) The time table showing the points of image acquisitions and reversal changes of thermal gradients. (B - D) The sliding window histograms showing the changes in distribution of a total of 15 worms migrating on the agar surface for the reversal changes of thermal gradients. Here, the window sizes were $5 \mathrm{~mm}$ at $2.5 \mathrm{~mm}$ intervals. The $\Delta T_{\text {steep }}$ was about $4.3^{\circ} \mathrm{C} / \mathrm{cm}$ and the green dotted lines indicate the locations of $T_{\mathrm{s}}$ formed on the agar surface.

the middle of an agar surface in the absence of the thermal gradient (red symbols in Fig. 4A). Thus, the population of worms had a Gaussian form as in the histogram of Fig. 4B. With a supply of DC power at $t=0$, a thermal gradient was established and the $T_{\mathrm{s}} \sim 22^{\circ} \mathrm{C}$ was formed at the location of $x$ $\sim 21 \mathrm{~mm}$ (green area in Fig. 4A).

A dozen worms placed in the region of warmer temperatures than $T_{\mathrm{s}}$ (right side of $T_{\mathrm{s}}$ in Fig. 4A) started to migrate down the gradient (right to left direction in Fig. 4A) using the cryophilic thermotaxis. After passing the temperature zone of $T_{\mathrm{s}}$, they stopped their thermotactic behavior and instead showed a random migration to the side of colder temperatures (left side of $T_{\mathrm{s}}$ in Fig. 4A) with other worms placed already on the cold side (also, see Fig. S5 in Supporting Information). Due to such random migration limited to the colder side, the peak location in the histogram of Fig. 4C was shifted to the left cold side, and their population also became broadened as $t$ increased (Fig. 4D).

\section{C. elegans accumulation induced by a thermal stimulus of} gradient-reversal

As mentioned above, the thermophilic migration in $C$. elegans requires well-controlled temperature conditions. As shown in Fig. 4, the population of worms on an agar surface with a steep thermal gradient appears dispersed on the side of colder temperatures, making the linear thermal gradient device not practical for worm-sorting or -collecting purposes. ${ }^{27}$ In this study, we suggest a strategic approach to utilize a gradientreversible temperature device for the effective collecting of the worms near $T_{\mathrm{s}}$.

As described in Fig. 4, the worms pre-incubated at $22^{\circ} \mathrm{C}$ were plated in the middle of an isothermal agar surface (Fig. 5B). At $t=0$, a DC voltage was applied to the Peltier modules to generate a thermal gradient. Once the linear gradient was established, some worms on the warmer side (right side of $T_{\mathrm{s}}$ in Fig. 5B) directly migrated toward $T_{\mathrm{s}}$, while others on the colder side migrated randomly (left side of $T_{\mathrm{s}}$ in Fig. 5B). Thus, the peak shift in their population was observed to the left, as shown in the histogram of Fig. 5C. At $t=30 \mathrm{~min}$, a thermal stimulus of gradient-reversal was applied by oppositely connecting the electrical polarity to each Peltier module. According to our measurement, the gradient shape was completely reversed within $10 \mathrm{~min}$ later and then reached a steady state within 20 min after changing the electrical polarity (Fig. S2B in Supporting Information). Despite the fact that most of the worms experienced a sudden temperature shock by gradientreversal, they recovered the cryophilic capability toward $T_{\mathrm{s}}$. Soon, most worms that were initially dispersed to the left side of $T_{\mathrm{s}}$ (before cold side) started to migrate along the newly generated negative gradient from the left to the right side as the temperature was higher, the opposite direction to the previous thermal gradient. With a further increase of $t$, the population of worms shifted to the right side of colder temperatures (Fig. 5D). To accumulate the population density near $T_{\mathrm{s}}$, we again reversed the direction of the thermal gradient at $t=55 \mathrm{~min}$. After $10 \mathrm{~min}$ later $(t=65 \mathrm{~min})$, a large portion of the worms had accumulated in a narrow temperature zone of $T_{\mathrm{s}}$ (Fig. 5E).

Such accumulation near $T_{\mathrm{s}}$ seems to be mediated by a large difference in thermotactic responses for both gradient directions: the fast directed migration along a negative gradient and the slow diffusive migration as illustrated in the schematic of Fig. 6. Initially, the worms are plated in a Gaussian shape with a peak in the middle of an isothermal agar surface (Fig. 6A). With a linear thermal gradient, the peak location is shifted to the cold side as most of the worms placed on the warm side (right of $T_{\mathrm{s}}$ in Fig. 6B) directly migrate toward $T_{\mathrm{s}}$ and then lose their directionality after reaching $T_{\mathrm{s}}$ (Fig. 6B). Compared to the slow dispersion by a diffusive migration of the worms on the colder side, the aggregation by a directed migration toward $T_{\mathrm{s}}$ is much faster (compare the magnitudes of arrows drawn in the schematic of Fig. 6C). To collect the worms further, some of the worms dispersed on the cold side near $T_{\mathrm{s}}$, the direction of a thermal gradient is reversed once more (Fig. 6D). As temperatures become higher than $T_{\mathrm{s}}$ on the previous cold side (right side of $T_{\mathrm{s}}$ in Fig. 6D), these worms begin to directly migrate along the newly formed negative gradient toward $T_{\mathrm{s}}$. Although on the opposite side of cold temperatures some of the worms are dispersed, it is not significant to the aggregation near $T_{\mathrm{s}}$. As a result, by repeating the process of gradient-reversal, the population of worms near $T_{\mathrm{s}}$ becomes higher with a sharp peak at $T_{\mathrm{s}}$ (Fig. 6D). However, such aggregation near $T_{\mathrm{s}}$ by the repetition of gradient reversal is a transient phenomenon because the sharp Gaussian distribution is slowly dispersed to the colder temperature side as $t$ increases.

\section{Conclusions}

In conclusion, we successfully demonstrated that the thermal 
A

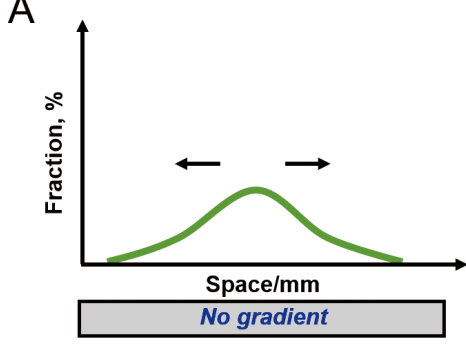

C

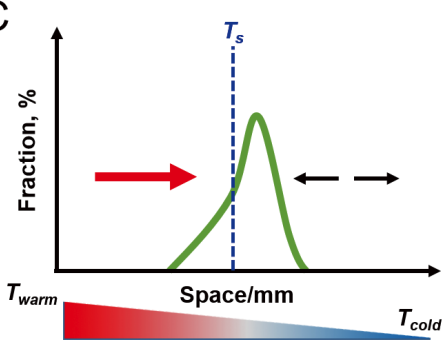

B
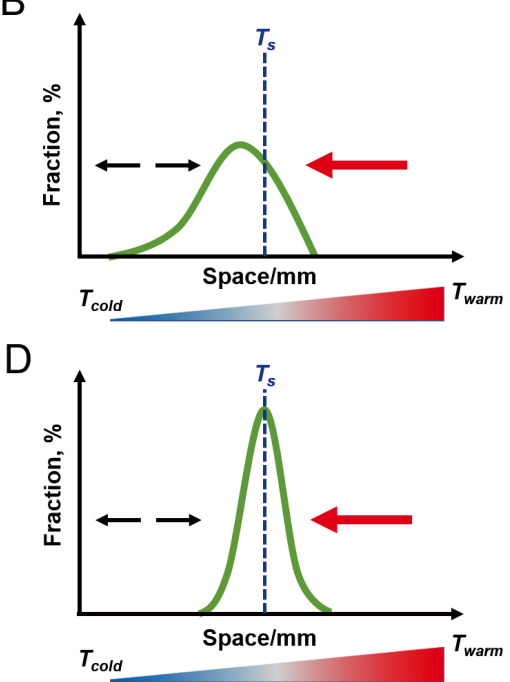

Fig. 6 Schematic illustrations showing a possible mechanism for aggregation of C. elegans as a reversal of a steep thermal gradient: (A) The initial distribution of C. elegans plated around the middle of an agar surface without a thermal gradient. (B) The left-shift in the peak location of the distribution with a broadness on the side of colder temperatures as a linear thermal gradient is applied. (C) The directed migration of worms in a reverse way due to a thermal stimulus of gradient-reversal. (D) The accumulation of worms in a narrow area of $T_{\mathrm{s}}$ as a gradient-reversal once more. Here, the black and red arrows indicate the Brownian motion in isothermal or the side of colder temperatures and the directed migration in the side of warmer temperatures, respectively. Also, the magnitude of arrows indicates the strength in the directed migration.

stimulus of gradient-reversal could be used as a triggering source to accumulate worms near the specific area of the desired temperature, as an alternative methodology distinguishable from the chemotactic assay used to study the social feeding behavior of worms. ${ }^{28-30}$ Despite a significant thermal shock by gradientreversal, C. elegans soon recovered the cryophilic ability to direct their migration toward $T_{\mathrm{s}}$. A thermal variation from a variety of environments has a major impact on physiological behaviors of C. elegans including growth rate, longevity, reproduction, and navigation. Therefore, our thermal stimulus device has a potential to be utilized extensively in various applications such as sorting and collecting systems for a variety of small living organisms that feature any thermal responses.

\section{Acknowledgements}

We thank Dr. U. Ko for assistance in experimental setup. This work was funded by Basic Research Funds from the Department of Mechanical Engineering at KAIST (A0601003029).

\section{Supporting Information}

Growth status of C. elegans used in our experiments (Fig. S1), temporal variation of a linear gradient during its transient time and the temperature stability in a steady state (Fig. S2), random migration of C. elegans on an isothermal plate (Fig. S3), isothermal tracking behavior of C. elegans (Fig. S4), additional data of a biased $C$. elegans thermotaxis reproduced in a steep thermal gradient (Fig. S5), and detailed description of $C$. elegans preparation are located in Supporting Information. This material is available free of charge on the Web at http://www.jsac.or.jp/ analsci/.

\section{References}

1. J. T. Pierce-Shimomura, B. L. Chen, J. J. Mun, R. Ho, Raman Sarkis, and S. L. Mclntire, Proc. Natl. Acad. Sci. U. S. A., 2008, 105, 20982.

2. A. Vidal-Gadea, S. Topper, L. Young, A. Crisp, L. Kressin, E. Elbel, T. Maples, M. Brauner, K. Erbguth, A. Axelrod, A. Gottschalk, D. Siegel, and J. T. Pierce-Shimomura, Proc. Natl. Acad. Sci. U. S. A., 2011, 108, 17504.

3. S. Jung, Phys. Fluids, 2010, 22, 031903.

4. J.-S. Park, D. Kim, J. H. Shin, and D. A. Weitz, Soft Matter, 2016, 12, 1892.

5. Y. Zheng, P. J. Brockie, J. E. Mellem, D. M. Madsen, and A. V. Maricq, Neuron, 1999, 24, 347.

6. L. Luo, N. Cook, V. Venkatachalam, L. A. MartinezVelazquez, X. Zhang, A. C. Calvo, J. Hawk, B. L. MacInnis, M. Frank, J. H. R. Ng, M. Klein, M. Gershow, M. Hammarlnd, M. B. Goodman, D. A. Colón-Ramos, Y. Zhang, and A. D. T. Samuel, Proc. Natl. Acad. Sci. U. S. A., 2014, 111, 2776.

7. T. Sugi, E. Okumura, K. Kiso, and R. Igarashi, Anal. Sci., 2016, 32, 1159.

8. C. I. Bargmann and H. R. Horvitz, Neuron, 1991, 7, 729.

9. D. R. Albrecht and C. I. Bargmann, Nat. Methods, 2011, 8, 599.

10. X. Wang, R. Hu, A. Ge, L. Hu, S. Wang, X. Feng, W. Du, and B.-F. Liu, Lab Chip, 2015, 15, 2513.

11. B. Han, D. Kim, U. H. Ko, and J. H. Shin, Lab Chip, 2012, $12,4128$.

12. A. Ward, J. Liu, Z. Feng, and X. Z. S. Xu, Nat. Neurosci., 2008, 11, 916.

13. E. M. Hedgecock and R. L. Russell, Proc. Natl. Acad. Sci. U. S. A., 1975, 72, 4061. 
14. I. Mori and Y. Ohshima, Nature, 1995, 376, 344.

15. A. Mohri, E. Kodama, K. D. Kimura, M. Koike, T. Mizuno, and I. Mori, Genetics, 2005, 169, 1437.

16. P. A. Garrity, M. B. Goodman, A. D. Samuel, and P. Sengupta, Genes Dev., 2010, 24, 2365.

17. C. A. Chi, D. A. Clark, S. Lee, D. Biron, L. Luo, C. V. Gabel, J. Brown, P. Sengupta, and A. D. T. Samuel, J. Exp. Biol., 2007, 210, 4043.

18. W. S. Ryu and A. D. T. Samuel, J. Neurosci., 2002, 22, 5727.

19. M. Beverly, S. Anbil, and P. Sengupta, J. Neurosci., 2011, 31, 11718.

20. Y. Yamada and Y. Ohshima, J. Exp. Biol., 2003, 206, 2581.

21. P. Jurado, E. Kodama, Y. Tanizawa, and I. Mori, Genes Brain Behav., 2010, 9, 120.

22. K. Nakazato and A. Mochizuki, J. Theor. Biol., 2009, 260, 56.
23. X. Wang, R. Hu, A. Ge, L. Hu, S. Wang, X. Feng, W. Du, and B.-F. Liu, Lab Chip, 2015, 15, 2513.

24. Y. Yan, L. F. Ng, L. T. Ng, K. B. Choi, J. Gruber, A. A. Bettiol, and N. V. Thakor, Lab Chip, 2014, 14, 4000.

25. S. Yoon, H. Piao, T. J. Jeon, and S. M. Kim, Anal. Sci., 2017, 33, 1435.

26. L. Luo, D. A. Clark, D. Biron, L. Mahadevan, and A. D. T. Samuel, J. Exp. Biol., 2006, 209, 4652.

27. D. Ramot, B. L. Maclnnis, H. C. Lee, and M. B. Goodman, J. Neurosci., 2008, 28, 12546.

28. M. de Bono and C. I. Bargmann, Cell, 1998, 94, 679.

29. A. B. Artyukhin, J. J. Yim, M. C. Cheong, and L. Avery, Sci. Rep., 2015, 5, 10647.

30. T. Sugi, H. Ito, M. Nishimura, and K. H. Nagai, Nat. Commun., 2019, 10, 683. 